\title{
Multi-modal management actions for public transport disruptions An agent-based simulation
}

\section{Conference Paper}

Author(s):

Siegrist, Mahsa (D); Ghandeharioun, Zahra (D); Kouvelas, Anastasios (1); Corman, Francesco (1)

Publication date:

2021-06

Permanent link:

https://doi.org/10.3929/ethz-b-000501419

Rights / license:

In Copyright - Non-Commercial Use Permitted

Originally published in:

https://doi.org/10.1109/MT-ITS49943.2021.9529305

Funding acknowledgement:

180545 - NFS Dependable Ubiquitous Automation (SNF) 


\title{
Multi-modal management actions for public transport disruptions: an agent-based simulation
}

\author{
Mahsa Rahimi, Zahra Ghandeharioun, Anastasios Kouvelas, Francesco Corman \\ Institute for Transport Planning and Systems \\ ETH Zurich, Stefano-Franscini-Platz 5, 8093, Switzerland \\ \{rahimim, zahrag, kouvelas, corman\}eethz.ch
}

\begin{abstract}
In a public transport disruption, management of the disruption plays a significant role in reducing passengers' inconvenience. This research aims to evaluate how the delay that passengers experience in a public transport disruption can be reduced, using multi-modal management actions. We consider various traffic management actions implemented in real-time to mitigate the downsides of a disruption, including route and mode adjustments, and capacity increase. Transport operators can implement disruption management actions, such as assigning more vehicle capacity/frequency to the running services; and can inform passengers about the disruption. The benefits of providing updated information to mitigate the downsides of the disruption are large, and could possibly lead to a better mode and route choice. The simulation of the impacts and dynamics of a reallife disruption in our study is performed by an agent-based framework. We demonstrate how much delay could be avoided by implementing multi-modal management actions and analyzing the extent to which they change passengers' travel behavior, in terms of travel mode and travel time. Results indicate that employing multi-modal management can lead to a reduction of $18 \%$ in the delay of affected passengers, when information and capacity management are used together.
\end{abstract}

Index Terms-disruption management, public transportation disruption, agent-based simulation

\section{INTRODUCTION}

Public transportation plays a major role in the daily life of the people. Disruptions in public transport (PT) have a negative impact on passengers' comfort and satisfaction. Disruptions can vary from minor incidents, such as delays due to adverse weather conditions, over-loaded vehicles, to the trains' breakdowns. Transport operators use disruption management actions to reduce the delay that passengers experience. Several actions can be used to overcome a disruption, such as updating the timetable, rescheduling, rolling stock, and increasing the running services' capacity, from an operator point of view; and informing passengers, so that they can adjust their mode and route choice. We use the term multi-modal disruption management actions when various real-time actions are implemented in managing PT disruptions to find the best feasible approach to reduce the delay and travel time. Among multimodal disruption management actions, informing passengers as a solution is very attractive, as it does not need massive investments in the infrastructure, vehicle, or personnel resources. The delay varies according to the time passengers become aware of the disruption and to the extent, they can re-plan their desired trips and activities. Therefore, it is in the interest of passengers and public transport service operators to examine and assess to what extent multi-modal disruption management actions have the potential to reduce the delay experienced by traveller; and to estimate any change in passengers' behavior throughout the disruption. We analyze the effects of such actions on passengers' delay, travel time, and the effects on travel mode choices. For simulating real life scenarios, we have implemented an agent-based simulation approach. For our simulation, we use a calibrated disaggregated demand model, which determines input for the agent-based simulation.

\section{PROBLEM DESCRIPTION AND CONTRIBUTION}

We assume a disruption is an event, mostly unexpected or unknown until shortly before, or even after its occurrence, which prevents some PT services from running as planned. Possible disruptions can be related, for instance, to operational limitations, failures, accidents, adverse weather conditions, etc.

Various approaches have been implemented to address the problem of disruption management in the public transportation context. In the current work we only mention the most recent research in this field. [1] studied railway disruption management action and proposed a MILP model that integrates passenger reassignment approach with timetable rescheduling. Providing real-time information studied in [2]-[4], helps travelers to react accordingly and adjust their travel plan to the updated timetable, notwithstanding the disruption and facing large delays. However, their studies focused only on informing passengers to reduce the adverse effects of PT disruptions while not considering increasing capacity as a disruption management action. PT operators can increase the transport capacity (longer vehicles or more frequent services) of existing lines, which can help to bypass the disruptive event [5]. Such efforts can all be included in the concept of a disposition timetable, which differs from the planned timetable. Determining a practical disposition timetable is a complex task to be quickly performed with an only approximate understanding of how the passengers might redistribute within the network and/or react to the new timetable [6]. Besides, some previous research quantitatively evaluates the disruption management practices on already occurred disruption [7]. The behavior of passengers is typically estimated based on multi-modal assignment techniques. User equilibrium solution refers to a situation in which through a day-to-day (where each iteration corresponds to one day) process, travelers, for instance, learn 
that some public transport lines are slower or faster and that some are close to capacity at a certain time and the travelers might get denied boarding, therefore, experiencing longer travel times. The final solution is an optimum response to the dynamic characteristics of the network. Non-equilibrium is a non-iterative approach which is appropriate for the simulation of unexpected events, in a non equilibrium state the passengers have not reached the most efficient plan in the network by iteration. Practically, there is insufficient empirical data available from past experienced disruptions to replicate them and study their impacts in simulation models [8]. Instead, a simulation can assist in choosing effective actions in real life, by means of an analysis of their respective results, including quantifying delays and associated monetary values for passengers and the whole PT network.

To the best of our knowledge, this research is the first attempt to bring various multi-modal management actions of disruption in a unified framework, and compare their impacts by analyzing their effects on passengers' behavior. Besides, we investigate and compare each of our scenarios in both equilibrium and non-equilibrium conditions. Therefore, We implement a within-day replanning approach for simulation of such unexpected disruptions. The results of this research are valuable for PT authorities for further understanding of passenger flow evolution after a disruption on a PT link, and to understand the risks and opportunities of disruption management actions to improve system performance in case of a disruption in PT networks. Our main contributions to the literature are: Firstly, We implement operational multi-modal management actions on a comprehensive set of scenarios (thirteen scenarios). We consider both passengeroriented actions such as providing information in real-time, enabling route and mode adjustment; and supply-oriented actions such as increasing the vehicle capacity or frequency of the running services. Secondly, We simulate thirteen scenarios with equilibrium/non-equilibrium conditions, reflecting expected/unexpected disruptions. We compare their impacts on passengers' delays and associated monetary value of time.

\section{Methodology}

\section{A. Agent-based simulation approach}

To simulate the response of travelers, and estimate their delay, we use an activity-based, agent-based simulation approach. The agent-based simulation analyzes individual behavior by modeling each traveler as an individual agent who makes his or her own decisions according to predefined rules. Agents interact with each other in the simulation environment where one agent's choice affects another agent's choice and, finally, the whole environment. The present research uses the software MATSim, which is based on an iterative procedure to approximate the day-to-day user equilibrium state [9]. Eventually, an equilibrium state is reached where the agents cannot further improve their plans unilaterally.

A daily-plan, which is one of the outputs of MATSim, is the actual sequence of activities and trips. For agent $a$ the daily plan $s_{a}$ includes a sequence of activities $(A c)$ and trips $(T r)$ to reach those activities. Equation 1 explains that the daily plan includes $i$ trips $(T r)$ and $i+1$ activities $(A c)$. For a simple example, agent $a$ can start in the morning by traveling from home $\left(A c_{1}\right)$ to work $\left(A c_{2}\right)$ by bus $\left(T r_{1}\right)$ and in evening returns home $\left(A c_{3}\right)$ by bus $\left(T r_{2}\right)$.

$s_{a}=\left(A c_{1}, T r_{1}, A c_{2}, T r_{2}, \ldots, A c_{i}, T r_{i}, A c_{i+1}\right)$

where $A c_{i}=\left(l_{i}, t_{\text {start }, i}, t_{\text {end }, i}\right), \operatorname{Tr}_{i}=\left\{\left(l_{\text {from }, i}, l_{\text {to }, i}, t_{i}, v_{i d}\right)\right\}$,

$v_{i d} \in V$

Each activity is defined by 3 elements of the location $\left(l_{i}\right)$ in which the activity happens, the time that the activity starts $\left(t_{\text {start }, i}\right)$ and the time that activity ends $\left(t_{e n d, i}\right)$. Each trip is also defined by 4 elements: location in which the trip starts $\left(l_{\text {from }, i}\right)$, the location in which the trip ends $\left(l_{t o, i}\right)$, the time that the trip starts $\left(t_{i}\right)$, and the PT vehicle $v_{i d}$ is used for traveling. Set $V$ includes the ids of all transit services.

\section{B. Within-day replanning approach}

The concept of user equilibrium, typical of transit assignment, and also implemented in basic Matsim version is not capable of modeling travelers' actions under disruptions [10]. As disruptions are not recurrent, travelers cannot learn from past experiences, and a day-to-day process looks illogical. To this end, a within-day replanning approach simulates only a single iteration, preventing problems resulting from an iterative simulation process [10]. For our simulation, we use a calibrated disaggregated model of demand (already used and tested in [2], which determines input for the agentbased simulation (also calibrated/ evaluated in many previous studies). In our simulation, we also use the module for withinday replanning developed by [4], which enables rerouting based on the updated disposition timetable; and considers the capacity of vehicles as a further constraint.

\section{CASE STUDY OF ZURICH CITY}

We evaluate the effects of a disruption on a large multimodal network; we chose Zürich and its PT network. However, the proposed methodology is not limited to any particular geographical situation and can be applied universally. We represent the population of Zürich utilizing agents, at a $1 \%$ sampling rate, that is, each agent represents 100 persons in real life; capacities of road links and vehicles are scaled accordingly, results in 12'072 agents. The disruption considered is the closure of a major railway link in the city, and it is confined to geographical (stations of Hardbrücke, Oerlikon, Zurich HB and Wipkingen) and time dimensions (16.00 to 19.00). The dotted/solid lines in Fig. 1 represents the disrupted/undisrupted rail services. Referring to this disruption, we identify agents affected by the disruption as those traveling at the disrupted time, on a disrupted vehicle, between the disrupted stations, in the normal day. In the simulated test case, 140 agents (therefore corresponding to 14'000 people in real life) cannot perform their usual trip; we identify them as "directly affected agents". As the disruption occurred in a specific part of the Zürich network, not all the agents in the whole city experience 


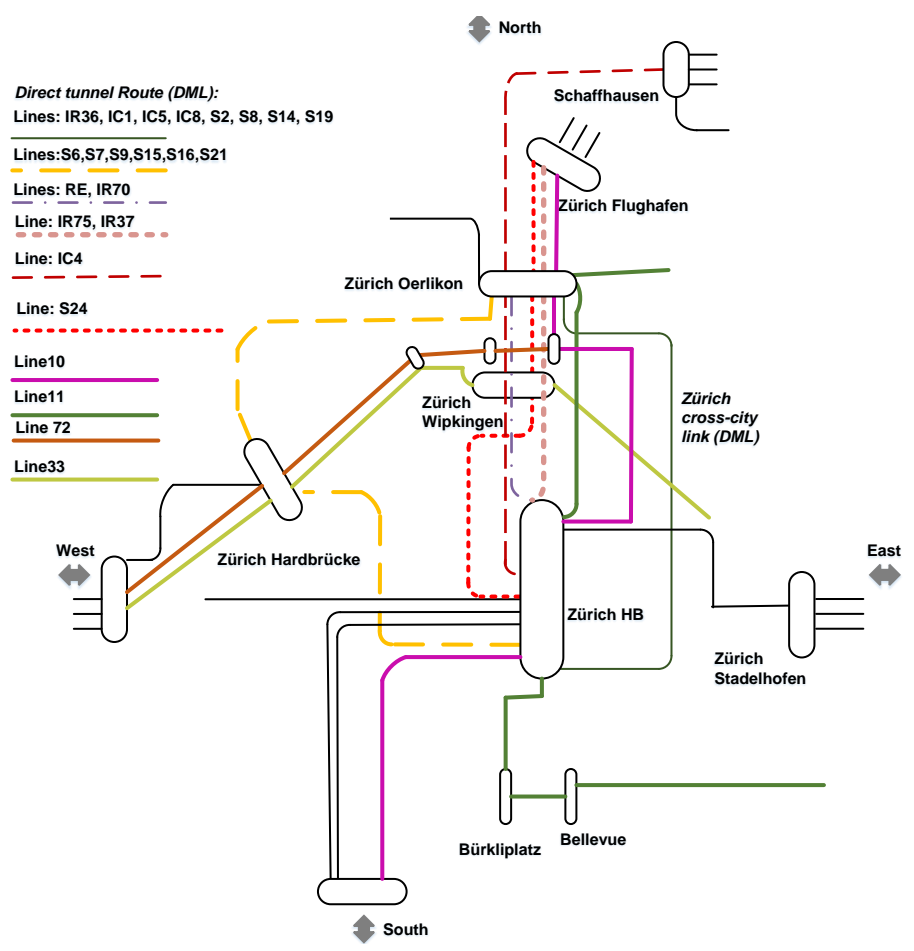

Fig. 1: Details of rail services (dotted color: disrupted; black solid: normal) and tram services bypassing the disruption (solid color), in Zürich network for the considered scenarios.

the disruption and its consequences. When considering all the 12072 agents, the effect will be relatively small. On the other hand, the 140 directly affected agents will suffer from substantial delays, ranging from a few minutes to hours delay time, depending on the circumstances of the scenario that we simulate. In the following, we present mode choice analysis for both cases, of all agents, and directly affected agents.

\section{Multi-modal DisRuption management actions}

We simulate scenarios based on three multi-modal disruption management actions: information management enabling mode and route adjustment, increasing vehicle capacity, and increasing the frequency of running services.

1) Information management action: In an unexpected disruption, the passengers' behavior relies on the information they receive in content and time. We explain the related scenarios for information management and define them based on our simulation criteria in the following scenarios: 1)"Normal day" is an equilibrium without the disruption scenario (base scenario, used for benchmarks). 2)"Equilibrium+disruption" is the equilibrium scenario with the disruption. Agents adapt their daily plans through the iterations, having the possibility to change mode, route, and departure times,reaching a new equilibrium. 3)"WithinDaySD" is a within-day replanning scenario in which all agents have information about the disruption when disruption starts. Agents can only change route in the PT network, but cannot change mode anymore. 4)"WithinDayST" is a within-day replanning scenario in which agents have information about the disruption at different times depending on the time they intend to perform their trip. In other words, agents are informed at start time of their trip about the disruption if their trip is in the time of disruption. Agents can only change route in the PT network, but cannot change mode anymore. 5)"No Information" agents do not have any information about the disruption. Their reaction consists of waiting at the train station until the next vehicle's arrival of the PT line they intended to take. Agents can only take later services, in the same route as originally planned.

2) Vehicle capacity management action: One alternative action in disruption management is increasing the capacity for running services. We present a scenario 6)"Line10-Large", which is a disruption scenario, combined with the information strategy equivalent to the "Start of the disruption" plus increasing the vehicle capacity of the tram line 10 by a large amount (100\%).And, similarly for bus line 72 , determining another scenario as 7)"Line72-Large". We analyze the most used PT line by directly affected agents, tram line 11 and we determine another scenario as 8)"Line11-Large". For further analysis, we increase the vehicle capacity of the whole PT network in Zürich city, for the entire day, and we simulate the following scenarios: 9)" WithinDaySD+Medium", 10)"Equilibrium+Medium ", 11)"WithinDaySD+Large", 12)"Equilibrium+Large". The scenarios "WithinDaySD+Medium" and "WithinDaySD+Large" include the information management action equivalent to the scenario "WithinDaySD", that is route adjustment, no mode adjustment. We simulate two levels of increase in the vehicle capacity of running services: the Large increase $(100 \%)$, and Medium increase $(40 \%)$, which is the minimum increase possible, given the sampling rate used in the agent based scenario. The scenarios "Equilibrium+Medium" and "Equilibrium+Large" combine those two vehicle capacity increase with the equilibrium condition: agents can change mode route and departure time choice.

3) Frequency management action: We consider one scenario of increasing the frequency (vehicle departures per hour) of a running PT service, to test whether higher frequency in the PT network yields better service variations considering agents' delay. To this end, we propose the following scenario: 13)"Line10-Frequency". According to the original timetable, line 10 has an average headway of 5 minutes in the PT network, however the frequency of running services is very variable throughout the day. In our scenario, the frequency of the line 10 is doubled, meaning that line 10 runs with an average headway of 2.5 minutes, with consideration of variation of headway depending on the time of the day.

\section{RESUlTS}

\section{A. Delay analysis, directly affected agents}

We compare the delay that directly affected agents experience in each scenario in Table I. We consider the delay for those activities planned in an agent's daily plan after the disruption start time (after 16 o'clock) until the end of the day, compared with the same activities in the base scenario. This delay is labeled as activity-delay. The base scenario, i.e. the 
equilibrium in a normal day, is the reference for calculating the activity delay; therefore, the amount of activity-delay is equal to zero minutes for this scenario. We report the average delay, and its 90 percentile. By having the total delays for agents, and the value of time, which is $27.81 \mathrm{CHF}$ per hour [11], and the sampling rate of the agents, we can estimate the monetary value of delay (in thousands of $\mathrm{CHF}$ ) for the entire set of travellers, which can be found in Table I. In Table I column "\% vs. *" stands for a percentage comparison between the scenario "Start of the disruption" and others. We add such a column to compare all multi-modal management actions with one specific information management action.

TABLE I: Delay analysis for 140 affected agents

\begin{tabular}{|c|c|c|c|c|}
\hline \multirow[t]{2}{*}{ Scenarios } & \multicolumn{2}{|c|}{$\begin{array}{l}\text { Activity-delay } \\
\text { (minutes) }\end{array}$} & \multirow[t]{2}{*}{$\%$ vs. (*) } & \multirow{2}{*}{$\begin{array}{l}\text { Value of delay } \\
(\mathrm{kCHF})\end{array}$} \\
\hline & average & $90 \%$ & & \\
\hline No information & 92.4 & 180.0 & 760.76 & 492.2 \\
\hline WithinDayST & 12.4 & 30.1 & 15.38 & 80.3 \\
\hline WithinDaySD (*) & 10.7 & 30.4 & 0.00 & 69.6 \\
\hline Equilibrium+disruption & 5.3 & 26.1 & -50.61 & 34.1 \\
\hline Line10-large & 10.6 & 30.4 & -0.93 & 68.9 \\
\hline Line72-large & 10.8 & 31.6 & 0.93 & 703.0 \\
\hline Line10-Frequency & 10.6 & 30.0 & -1.49 & 68.5 \\
\hline Line11-large & 8.8 & 27.9 & -18.17 & 56.9 \\
\hline WithinDaySD+Medium & 8.9 & 28.4 & -16.78 & 57.9 \\
\hline Equilibrium+Medium & 4.5 & 15.1 & -58.15 & 29.1 \\
\hline WithinDaySD+Large & 8.5 & 28.4 & -20.41 & 55.4 \\
\hline Equilibrium+Large & 3.0 & 15.1 & -71.95 & 19.5 \\
\hline
\end{tabular}

By comparing the delay value in the scenario "WithinDaySD+Medium" with the scenario "WithinDaySD" in Table I, it can be seen that if we increase the capacity of PT vehicles in the disruption situation by medium level, the average delay that directly affected agents experience is reduced by around $17 \%$.

Adding to the vehicle capacity of Line 11 (scenario "Line11-large") results in less delay for directly affected agents compared to increasing the capacity of other PT transit lines. Agents in scenario "Line11-Large" experience 8 minutes of delay, around $18 \%$ less than scenarios "WithinDaySD", "Line10-Large", "Line72-large" and "Line10-Frequency".

Table I also shows that if agents become aware of the disruption at the WithinDaySD, instead of when they want to start their trip, their average delay decreases by $15 \%$. Table I, it can be seen that informing agents about the disruption at the start time of the disruption can reduce the delay the same as the scenarios in which the vehicle capacity or frequency of lines mostly used by affected agents is increased. In the scenario "WithinDaySD," agents experience an average of 10 minutes delay, which is the same as delays in scenarios "Line10-Large" and "Line10-Frequency". Because changes in timetable and capacity of vehicles requires larger investments than provision of real-time information. We can conclude that the benefit of real-time information management action is equal to proposed infrastructure management actions in reducing delay with considerably lower costs. By increasing the capacity of vehicles in the PT network, the scenario "WithinDaySD+Large" yields to $20 \%$ reduction in the delay, compared to the scenario "WithinDaySD". This result is not far from what is achieved already with a medium increase, (reduction of 17\%). The same comparison can be extended to the monetary value of delay. Comparing "Line10-Large" scenario with "Line10-Frequency" scenario, the increased frequency yields less delay compared to increasing the capacity of vehicles (against larger resource costs, for the operators; those are not discussed here).

In Table I, it can be seen that increasing the capacity of PT vehicles by a large level can have quite the same impact on agents' delay (causes a reduction of $20 \%$ in the delay) as increasing the capacity of PT vehicles by a medium level (again, a reduction of $17 \%$ in the delay). However, increasing the whole PT network's capacity indeed requires enormous investment, for the PT infrastructure and services. Therefore, we can conclude that the benefit of a medium increase in the PT vehicle capacity can be as significant as a large increase in capacity, which would require an even larger investment.

\section{B. Mode choice and travel time, all agents}

Analyzing the mode choice is critical to understand how the different modes help to mitigate the consequences of a disruption. In this sense, it is interesting to evaluate how strategic management disruption actions influence the agents' behavior and their traveling pattern. In a within-day, nonequilibrium setting, when travelers receive the information, they look for alternative routes to complete their traveling. The travelers using the PT mode cannot promptly change the mode to a private car, as they have no vehicle available right away. The travelers can instead use the PT services, which are running despite the disruption; this might result in longer trips, and/or more transfers, and/or longer waiting times to reach their destination. Specifically, travelers will use PT services, which were not typically using (i.e., compared to the user equilibrium situation without disruption). In other words, passengers affected by the disruption, who cannot take their desired PT vehicles, have to board other PT vehicles. Therefore, we might hypothesize that we will not expect tangible differences in the mode choice in the unexpected (non-equilibrium, within-day) scenarios. The scenarios based on equilibrium conditions instead exhibit to some extent a mode shift, as they allow a route adjustment as a consequence of the disruption.

We consider here a subset of scenarios, namely the basic scenario as a reference, with, and without disruption; two scenarios based on information provision but without the possibility to perform route adjustment ("WithinDaySD", nonequilibrium, within-day), and combining information provision with vehicle capacity adjustments ("WithinDaySD+Medium"). Finally, we also analyse one disrupted scenario with vehicle capacity management action ("Equilibrium+Medium") with equilibrium, that is including route choice adjustment.

The mode choice analysis in this research is based on each trip that agents traveled throughout the whole day (before and after disruption time). We have four modes of traveling, PT, car, walk, and bike. The percentage of the mode 
choice statistics under different scenarios are shown in Fig. 2. The modes are differentiated with the colors. The order of scenarios from the biggest circle to the smallest circles are "Normal day", "WithinDaySD", "Equilibrium+disruption", "WithinDaySD+Medium", and "Equilibrium+Medium". We also analyze the travel time per each mode for directly affected agents, shown in Fig. 3. X-axis reports the scenarios' names, Y-axis reports the travel time (hours, sum over all agents) in one simulated day; the modes are differentiated with colors. We also illustrate the total travel time spent on all modes with the title "sum". Fig. 2 shows that among the mode choices

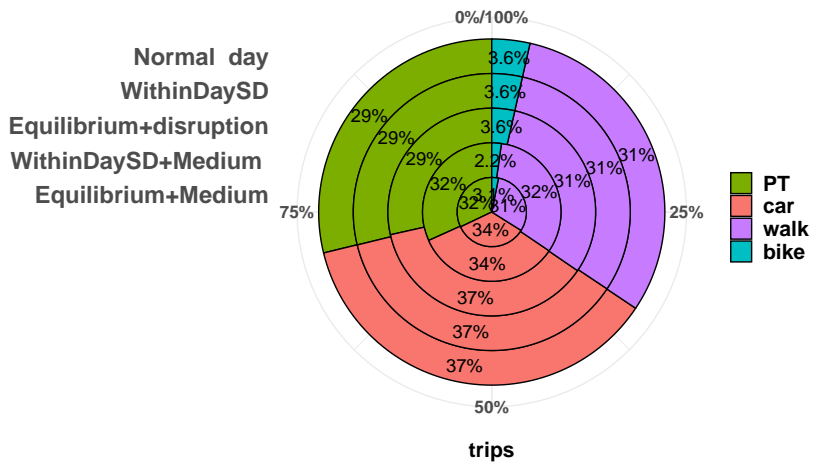

Fig. 2: Mode choice analysis, for all agents

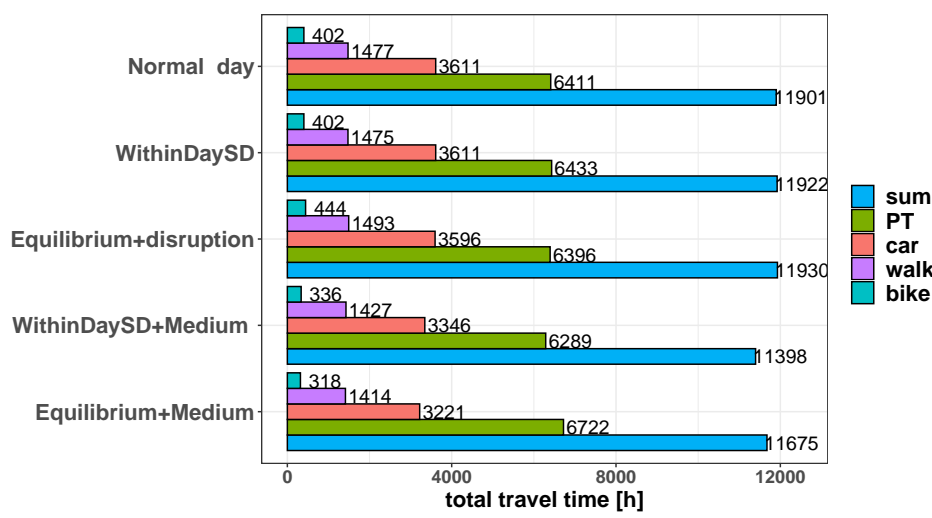

Fig. 3: Travel time spent per travel mode, for all agents

of traveling, car-mode is the most used mode of transport by the agents in all scenarios, followed closely by PT-mode. Agents in all scenarios spend more than half of their daily travel time on the PT-mode (Fig. 3). Such results indicate the importance of PT-mode in the daily-plan of agents and their dependency on PT-mode for commuting. After car mode, the most used travel modes are PT, walk, and bike with the lowest usage. By comparing "Normal day" (outermost circle) and "Equilibrium+disruption" (third circle) scenarios in Fig. 2 and Fig. 3, it can be seen that in those two user-equilibrium states, the disruption results in an overall decrease of PT-mode usage; and PT travel time. Instead, the usage of car-mode and walk-mode both increase slightly, identifying a modal shift. Comparing "Normal day" and "Equilibrium+Medium", we can see that a medium increase in the transit capacity of vehicles in the PT network, even in disrupted scenarios, yields to an increase of around 3\% in PT-mode choice; and a comparable increase in travel time by PT-mode; and a decrease around $2 \%$ in both choosing car-mode and the car travel time. PT-mode's highest travel time can be observed in the scenario "Equilibrium+Medium" and "WithinDaySD+Medium". In contrast, the lowest amount of travel time for car-mode is observed in the three scenarios. Such a result indicates that increasing the capacity of PT services by medium level directly affects the usage of PT-mode by the agents. Finally, the mode changes in the non-equilibrium scenario ("WithinDaySD") are not tangible, as already described before.

\section{Mode choice and travel time, directly affected agents}

In every disruption, some travellers are extremely affected due to the disruption of the PT services they use regularly, while, many travellers are partially affected. Where here investigate the effects of the disruption and management actions just on the 140 directly affected agents. This section is dedicated to quantifying the mode choice in scenarios for directly affected agents, similarly to the previous section. The statistics of the mode choice under different scenarios are shown in Fig. 4. The amount of travel time that directly affected agents spend on different modes is also presented in Fig. 5. Similar to the behavior of all agents in Fig. 2, the scenario "Equilibrium+disruption" has the lowest share of PTmode. However, directly affected agents use PT-mode by far more than any other travel modes; the same trend can be seen in travel time. This is obvious, as the disruption is on the public transport network, and directly affected agents must have been using the disrupted lines at some point through the disruption. By comparing "Normal day" and "Equilibrium+disruption"

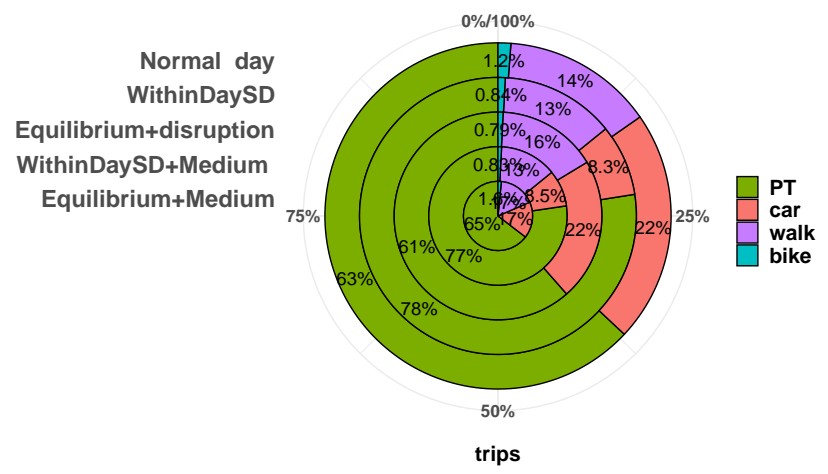

Fig. 4: Mode choice analysis, for 140 directly affected agents

scenarios, it can see that the disruption shifts directly affected agents towards the more usage of the car (22.2\% in "Equilibrium+disruption"), which is the highest rate of using car-mode by directly affected agents in all scenarios. Agents in "Normal day" also use PT-mode 2\% more compared with "Equilibrium+disruption". The highest share of PT-mode is seen in "WithinDaySD" and "WithinDaySD+Medium" whereas in Fig. 2 for all agents, the highest PT-mode share belongs to 


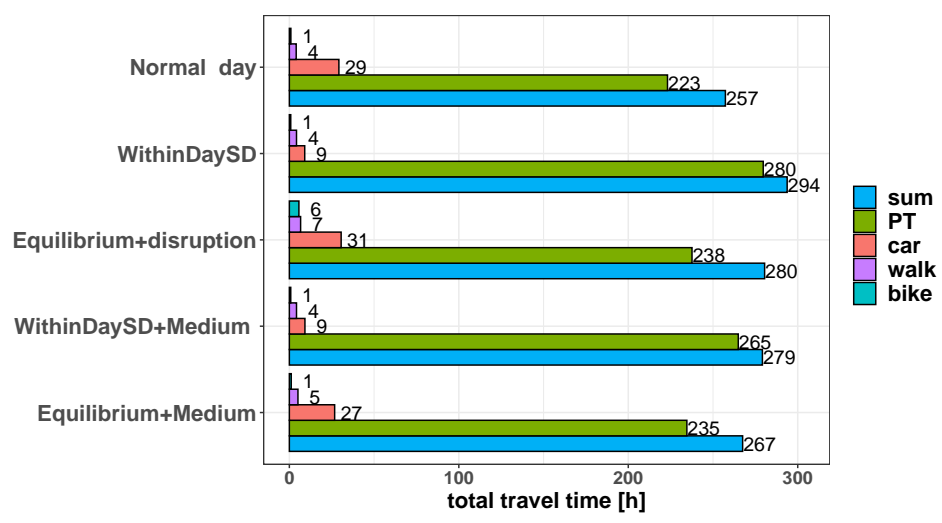

Fig. 5: Travel time spent per travel mode, for 140 directly affected agents

both "WithinDaySD+Medium" and "Equilibrium+Medium". The possible explanation for such observation is that directly affected agents have to reach their activity locations and perform their daily-plans, and when their direct trains is disrupted, they have to make it up by other PT services. Besides, when the vehicle capacity is increased, they can travel more freely. However, through the day-to-day iterations, they learn and adapt their daily travel plan to the disrupted situation, such as switching to car mode. It can be seen that directly affected agents use car mode $8.5 \%$ more in "Equilibrium+Medium" compared with "WithinDaySD+Medium". Fig. 5 shows that the lowest travel time is achieved in a "Normal day". In contrast, the "WithinDaySD" scenario has the largest travel time spent by the directly affected agents on all travel modes (showed as "sum" with blue color in Fig. 5). Besides, two increased-capacity scenarios, "WithinDaySD+Medium" and "Equilibrium+Medium" have the lowest amount of travel time. Therefore, although information strategy alone can considerably reduce the delay, implementing multi-modal management actions, informing agents, and increasing vehicle capacity can reduce the total travel time for the directly affected agents more effectively. A further evaluation should also include the associated resource costs for increasing the PT capacity as in the tested scenarios. The cost on the operator is difficult to estimate, depending on working conditions, processes, and internal agreements. For some scenarios, the operator costs might be more than the value of the time saved for passengers.

\section{CONCLUSION}

In this work, we evaluate the impacts of multi-modal management actions to manage disruptions in public transport networks, to reduce passengers' delay. We evaluated and compared the results of multi-modal management actions on passengers' behavior by analyzing the effects on passengers' delay and changes in travel mode and travel time spent. Among the multi-modal management actions, we considered realtime information provision action, enabling adjusting mode and route choice by the affected travelers, and capacity and frequency increase. Our results indicate that passengers' delay varies according to the time they become aware of the disruption. We have shown that providing information to passengers at the start time of the disruption can reduce the delay by an amount comparable to infrastructural management actions, such as increasing the vehicle capacity/frequency of some public transport line, which would require enormous investments. Moreover, increasing the vehicle capacity/frequency of lines is most effective only when targeting the specific lines, which are identified as the most critical for the directly affected passengers. An increase in the whole public transport vehicle capacity of running services might have limited benefit on a specific disruption; therefore it is important to use decision support to identify exactly which lines should have an increased capacity or frequency.

\section{ACKNOWLEDGEMENT}

Research supported by the Swiss National Science Foundation under NCCR Automation, grant agreement 51AU40_180545.

\section{REFERENCES}

[1] Y. Zhu and R. M. Goverde, "Integrated timetable rescheduling and passenger reassignment during railway disruptions," Transportation Research Part B: Methodological, vol. 140, pp. 282 - 314, 2020.

[2] N. Leng and F. Corman, "The role of information availability to passengers in public transport disruptions: an agent-based simulation approach," Transportation Research Part A: Policy and Practice, 2020.

[3] A. J. Khattak, X. Pan, B. Williams, N. Rouphail, and Y. Fan, "Traveler information delivery mechanisms: Impact on consumer behavior," Transportation Research Record, vol. 2069, no. 1, pp. 77-84, 2008.

[4] M. Rahimi and F. Corman, "Quantifying the heterogeneity of direct and indirect effects of a public transport disruption with agent-based simulation," 20th Swiss Transport Research Conference, 2020.

[5] J. Liang, J. Wu, Y. Qu, H. Yin, X. Qu, and Z. Gao, "Robust bus bridging service design under rail transit system disruptions," Transportation Research Part E: Logistics and Transportation Review, vol. 132, pp. 97-116, 2019.

[6] F. Corman, A. D'Ariano, A. Marra, D. Pacciarelli, and M. Samà, "Integrating train scheduling and delay management in real-time railway traffic control," Transportation Research Part E: Logistics and Transportation Review, 2016.

[7] D. Schipper and L. Gerrits, "Differences and similarities in european railway disruption management practices," Journal of Rail Transport Planning and Management, vol. 8, no. 1, pp. 42 - 55, 2018.

[8] M. Yap and O. Cats, "Predicting disruptions and their passenger delay impacts for public transport stops," Transportation, 2020.

[9] A. Horni, K. Nagel, and K. W. Axhausen, The Multi-Agent Transport Simulation MATSim. London: Ubiquity Press, 2016.

[10] C. Dobler and K. Nagel, Within-Day Replanning. London: Ubiquity Press, 2016, p. 187-200.

[11] K. W. Axhausen, S. Hess, A. König, G. Abay, J. J. Bates, and M. Bierlaire, "Income and distance elasticities of values of travel time savings: New swiss results," Transport Policy, vol. 15, no. 3, pp. $173-$ $185,2008$. 